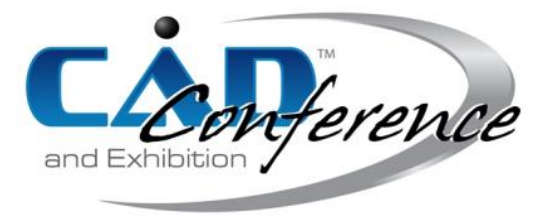

Title:

\title{
Haptic Feedback Glove for Arm Rehabilitation
}

Authors:

Natalia Rodriguez, natalia.rodriguez@mail.polimi.it, Politecnico di Milano

Matteo Sangalli, matteo4.sangalli@mail.polimi.it, Politecnico di Milano

Monika D. Smukowska, monikadorota.smukowska@mail.polimi.it, Politecnico di Milano

Mario Covarrubias, mario.covarrubias@mail.polimi.it, Politecnico di Milano

Keywords:

Haptic feedback, Arduino, Sharebot, Unity3D, leap motion and rehabilitation.

DOI: 10.14733/cadconfP.2021.303-307

\section{Introduction:}

The following document outlines the design process of a haptic feedback glove. The aim of this project is to give the user a haptic feedback once they are in contact with an object in a virtual interactive 3D space. In order to induce a haptic feedback, Unity3D is used to simulate the 3D virtual space, Leap motion is used in order to track the hands of the user and map them on the 3D virtual space. Finally, the signal from Unity is sent to Arduino which is able to produce the signal to initiate the vibration motors and led lights. The purpose of this technology is to help people with reduced motor ability, to perform physiotherapy exercises while playing an interactive game that involves haptic feedback.

Physiotherapy is a common treatment for people with reduced mobility, however nonattendance rates are high across the EU, this can be attributed to several factors; inability to afford the treatment, inability to reach the center or find the time for the session. Attending physiotherapy frequently is not feasible for some patients, therefore, most have to continue the treatment at home without guidance or way of verifying their progress.

Haptic feedback uses vibration as a communication tool when interacting in a virtual space. By using haptic feedback and hand tracking technology it is possible to indicate to the user (through vibrations) if the movement has been successfully achieved. Once the data has been stored it can indicate the progressive improvement of the persons mobility over time, which means both the patient and the physiotherapist can review useful evidence of their progress.

The main advantage of this technology is that it is affordable and portable which means that it can be recommended to those who are not able to go to a center to receive treatment and have to continue their recovery on their own.

\section{Design specifications:}

The purpose of this project is to help people with reduced mobility or a disability specific to the arm region with the aim of improving their mobility by introducing the possibility to perform self-guided virtual rehabilitation. Currently in EU for a population of 100000 people there are around 118 physiotherapist with increasing demand, this design aims to fulfill the gap in demand. [10]

The haptic gloved is aimed to a wide range population, from people that have suffered a stroke and need to regain motor ability, to those recovering from a surgery in such location or were born with a motor disability. For this reason, a principal aspect of the project was to ensure older age groups and 
younger age groups were able to equally use this product. Therefore, the design described is lightweight, economic, and ergonomic in order to ensure accessibility.

\section{Design process}

For the design we focused on simplicity and accessibility for the user. Thus, the model was inspired by the exoskeleton of the hand.

Velcro was proposed as a securing system as it allows a larger range of people to be able to utilize the product. Additionally, Velcro is an easy system that one person alone can manage to put the device on without assistance (depending on the level of their mobility), this is important as perhaps the rehabilitation of the patient will take place at home where this person might not be accompanied.

Design development (CAD)

The bracelet and fingers were designed in Inventor [4]. The size of each finger is taking into consideration to fit the average size of a person's hand. At the top of every finger there was a place for the motor and along the middle a place for the wires. Additionally, we designed thin covers for each finger to protect the motors from the environment.

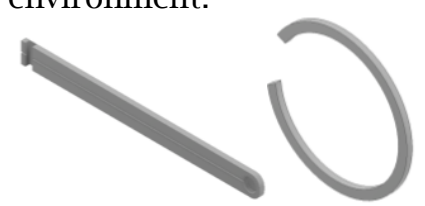

Fig. 1: First design of finger and bracelet.

Subsequently, we created a ring support for the Velcro as well for the finger as for the bracelet (Figure 2) in order to position the Velcro.

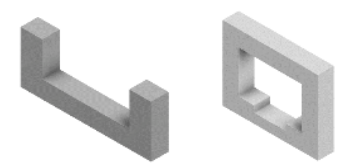

Fig. 2: Velcro supports.

Finally, due to a lack of space on the bracelet for the fingers, they were modified to be thinner as shown on the Figure 3. Additionally, the shape of the bracelet was also changed to a more comfortable one as shown below. Additionally, the top (cover) part of each finger was adequately modified, these modifications were done after evaluation of the initial prototype.
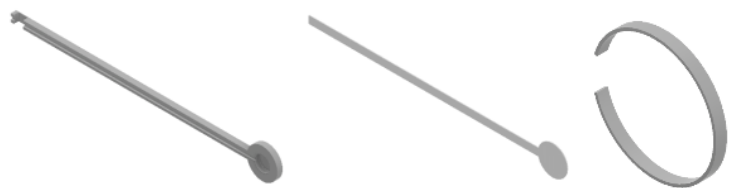

Fig. 3: Final design of the fingers and bracelet.

The elements were assembled in Inventor and the result can be seen on the Figure 5.

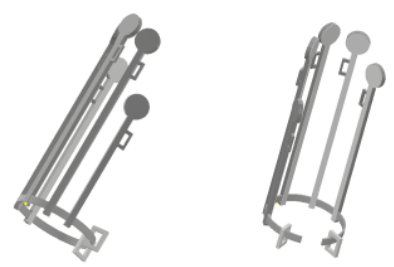

Fig. 4: Assembly in Inventor. 


\section{D Printing}

\section{Material choice}

The procedure for manufacturing is 3D printing, the recommended material for this purpose is TPU.

Thermoplastic polyurethane (TPU) is any of a class of polyurethane plastics with many properties, including elasticity, transparency, and resistance to oil, grease, and abrasion. Technically, they are thermoplastic elastomers consisting of linear segmented block copolymers composed of hard and soft segments [1]. This material is suggested primarily due to its elastic behavior which is need for this application.

\section{$3 D$ printing}

The designed parts in Inventor (Autodesk)[4] were prepared for the 3D printing. Firstly, they were exported to the .stl files, remembering to choose the correct units $(\mathrm{mm})$ and later they were imported to the Slic3r software. After choosing the correct settings the g-codes were created and put into the 3D printer which was the Sharebot Next Generation [5]. The whole printing procedure is depicted in the Figure 10 .

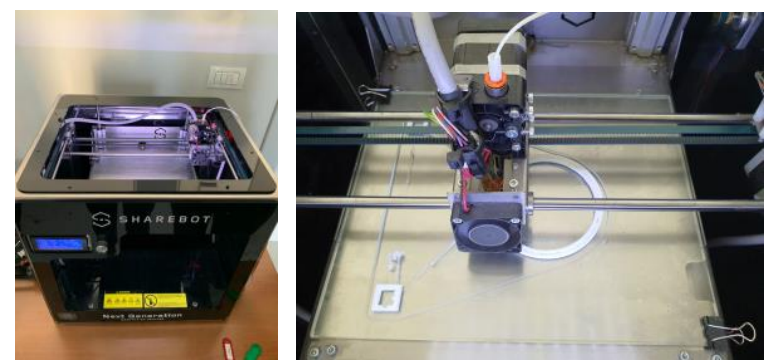

Fig. 5: (a) 3D-printer (b) printing the bracelet.

The physical assembly was composed of two mayor steps the first setting up the electronic circuit connected Arduino.

The soldering process had to be repeated several times, the light installed in the bracelet is a threetone led which means there are 4 wires which are connect to the Arduino circuit. Additionally, each finger has a vibration motor which means that there are two wires that are to be connected to the Arduino circuit.

The second step of the physical assembly was to put the circuit together with the 3D printer pieces. To assemble the pieces, it was necessary to first insert the motors in the 3D printed fingers then mount them on the bracelet and cover them with a layer of TPU. Once this process has been done for all the fingers hooks are placed at the bottom part of the fingers, to hold the Velcro in place.
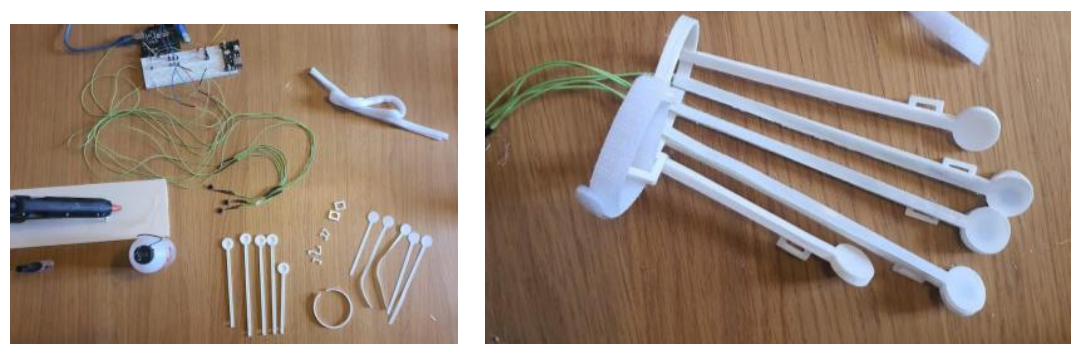

Fig. 6: (a) Set up for physical assembly (b) Final assembly of the haptic glove.

The result obtained should look like an exoskeleton which has all the wires coming out from one centralized point. This is to avoid the user getting tangled with the wire which can inevitably lead to rupture of the circuit. 
Unity3d and leap Motion:

We used unity Unity3D [7] to create an interactive platform for the user. Unity is program which allows the creator to generate, command and produce games amongst other features found within the application.

Unity3D contains prefabricated assets that are compatible with leap motion. This asset contains an interaction module which has defined different contact and collision within the game. This is especially important as it is in this module where the Arduino code will be installed. The aim of our project is to create an interface where the leap motion [6] controller can map the hand. Once the hand interacts with Unity3D interface a trigger is sent to the Arduino circuit [2] which will give the user the feedback.

In order to personalize the environment to fit our requirements we took two examples the Interaction object scene (interactive shapes) and the Basic UI scene (interactive buttons). In both cases we assigned colors to all shapes and buttons that can be interacted with. This color is respectively licked to the Arduino code that will activate the corresponding color of the haptic glove. Finally, when putting it all together we can create an interface that receives data from leap motion transfer into the virtual space in Unity3D and as able to transmit a signal to Arduino to simulate a haptic feedback.

\section{Arduino Circuit:}

To produce vibration (haptic feedback), we used mini motors that require 5V DC supply. This motor, such as all the type of motor, absorb a significantly level of current. Arduino is not able to supply all the needed current; therefore, to avoid Arduino failure. It is suggested to insert an external power supply. To control the motor, we use a Transistor command from Arduino. To control the transistor, we choose to use a PWM Digital Pin of Arduino to set a vibration value. This is an important aspect of the project as it allows the patient to adjust the settings of the haptic feedback.

The motors work for a few seconds every time that a patient touches a virtual object. This means that our circuit is under a significant stress. To avoid problem and circuit failure it is suggested to insert a diode. This will sustain the variation voltage, as it is able to discharge the voltage level. Finally, an interesting component is the digital pin as it will be ablet to give visual feedback to the user once it is in contact with an object.

Instructions of use:

In order to be able to fully use this program, there are some requirements the first is to have as a minimum two USB ports available and power source. Then it is required to download Unity (18 to latest versions compatible). It is additionally necessary to download Arduino interface and finally the leap motion controller adapter. All the programs mentioned are free source and can be downloaded from the internet.

To setup the device the first step is to connect the Arduino circuit to an electricity source then connect it to your computer the Arduino file can be found under Arduino carpet in Unity3D folder. Open this script and download it to the Arduino circuit it should make the lights of the LED and motors go off. Subsequently you must connect to leap motion to your computer. Once all the steps have been completed it is now simple to press play and enjoy the experience of haptic hand.

\section{Implementation:}

As part of this project, we had the possibility to work with ASPOC Association - Association for the Development of Cognitive Potential [3]. ASPOC is an association of parents of children with cognitive disabilities. They provided the possibility to introduce this technology to people with reduced mobility. The aim of the exercise was to introduce the technology and allow the people to perform the exercise with such game. The simplicity of the platform once installed allowed the users to quickly understand how to interact with the system. The haptic feedback allows the users to comprehend with better accuracy the placement of the object in the 3D environment. Finally, visual feedback made it look like a game which motivated the people to continue interacting with the objects hence performing the exercises. 


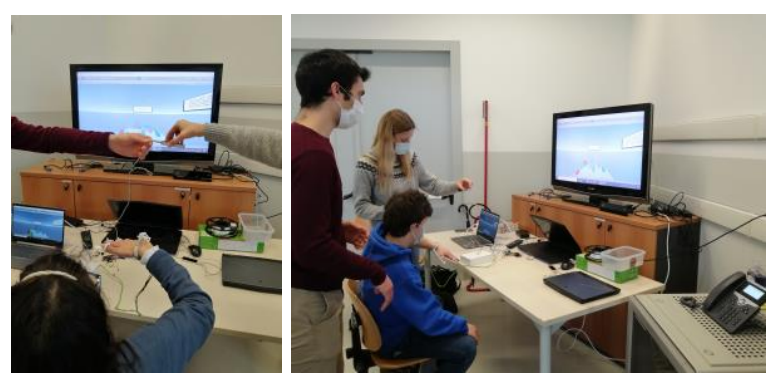

Fig. 7: Haptic feedback glove in use with members of ASPOC.

Conclusions:

This project included a design, manufacturing, assembling and game development of the haptic feedback glove for rehabilitation purposes. The design was chosen mainly due to its simplicity and fulfilling the stated constraints. 3D additive manufacturing was proposed as it is a cheap and fast way of prototyping.

In this work we developed a glove and a software to facilitate rehabilitation in patients that lost temporally their motor ability. This system has proven to be cheap and transportable, and it will allow patients and physiotherapist to remotely follow the users progress.

This project was developed considering several aspects, starting from make a system easy to manufacture, comfortable, ergonomic, and safe. The system was able to adapt to certain parameters to fit with the patient's necessity, such as the intensity of vibration and mobility restrictions, hence, the use of Velcro. These make our system versatile and able to be adapted to several cases of reduced mobility.

\section{$\underline{\text { References }}$}

[1] American Chemical Council: Thermoplastic Polyurethane, https://polyurethane.americanchemistry.com/Introduction-to-

Polyurethanes/Applications/Thermoplastic-Polyurethane/

[2] Arduino: https://www.arduino.cc/

[3] ASPOC - Associazione per lo Sviluppo del Potenziale Cognitivo, http://www.aspoc.it/

[4] Autodesk Inventor 2021: https://www.autodesk.com/

[5] Sharebot: https://www.sharebot.it/en/.

[6] Ultraleap: https://www.ultraleap.com/

[7] Unity: https://unity.com/

[8] Unity: Oculus: https://developer.oculus.com/unity/

[9] Vuforia: https://www.ptc.com/en/products/vuforia

[10] WHO: Indicator Practising physiotherapists per 100 000, 2016: https://gateway.euro.who.int/en/indicators/hlthres_195-practising-physiotherapists-per- $\quad 100$ $\underline{000 /}$ 